\title{
Simulation system development and application of slope or landslide
}

\section{model test}

\author{
DU Yue-wu ${ }^{1, a}$, YIN Xiao-tao ${ }^{2^{*}, \mathrm{~b}}$, \\ ${ }^{1}$ Applied Technology Collge of Dalian Ocean University, Dalian 116300, China \\ ${ }^{2}$ State Key Laboratory of Geomechanics and Geotechnical Engineering, Institute of Rock and Soil \\ Mechanics, Chinese Academy of Sciences, Wuhan 430071, China \\ a308585238@qq.com,byxt9406@163.com
}

\begin{abstract}
Key words: slope or landslide; failure mechanism; particle flow code; simulation system; model test.

Abstract: Numerical or model test is a main way of understanding failure mechanism of slope or landslide. Practical test is often limited by cost, time and difficulty in determination of material similarity ratio, which don't lie in numerical one. This paper develops a simulation system of slope or landslide virtual model test by matlab mixed fish language based on software of particle flow code. Physical and mechanical characteristics of different strata, substance construction, raw structure and human engineering structure are determined by trial computation of the system, which can be used to study relationship between macro and micro parameters of material and change of failure mechanism of slope or landslide caused by them. All these parts can built slope, landslide and collapse in practice and be used to study their reckoning mechanism, fail modes and laws of these disasters. The system has functions such as servo loaded and unloaded of different geometry models, tracing stress and strain information of any point or any step within models during test period, capturing energy transformation between boundary energy, kinetic energy, friction energy and strain energy during loading and unloading process and obtaining failure shape, micro crack propagation and number of different type of micro crack during experimental process. These can be used to study failure mechanism of slope or landslide affected by different influence factors.
\end{abstract}

\section{Introduction}

The slope and landslide problem is an old geotechnical engineering problem. Present methods for its stability evaluation include two categories, limit equilibrium method $[1,2,3]$ and strength reduction method [4,5,6,7], with safety coefficients and sliding surface as the evaluation parameters. Also there is a method of degree of reliability which is based on probability and contains the degree of reliability and failure probability. Most analyzing methods are numerical calculation, supervision and model test $[8,9,10,11,12]$. Numerical calculation has the problems of calculation scale and model accuracy balancing, characteristics of rock and soil structure modeling, mesh distortion and convergence $[4,5,6,7]$. Supervision can only reveal the stress and strain changing of the slope and its warning and forecasting is often empirical. Model test is costly and hard to simulate the material similarity ratio. Additionally, it is difficult for the indoor model to simulate the material and structural similarity simultaneously $[13,14,15,16]$.

Finite element has great difficulty to simulate the rock and soil damage mechanism and to model the structural characteristics of the rock and soil, e.g., traditional limit equilibrium method can hardly take the embedded part in the slip mass into account, and slope cannot contain concave geometry, neither the slope surface. Finite element can only calculate the large deformation 
condition. It has deficiency in reflecting the mechanical behavior of the rock and soil after peak strength, and is hard to reflect the dynamics characteristics of the kinetic and potential energy transforming.

This paper established a slope and slide test simulation system with the particle flow code. The structural factor of rock and soil can be considered in this system. The effect of the rock and soil macro and mesoscopic mechanics behavior, the material composition and the structural factor on the stability of landslide then can be explored. The dynamic equations of discrete element method can well reflect the kinetic-potential conversion, the slide surface forming from the slope movement rebalancing, the impact erosion and undercut in the foundation during the sliding.

\section{Framework of the slope and slide model test simulation system}

\section{Objective, applications and technical features}

Most researches of the rock or soil slope failure process are tests that include numerical and physical model tests. The main kind of the latter is centrifugal model test which is expensive and test amount is then limited. The experimental phenomena often are apparent observation which can hardly assort with monitoring equipment of loading and inner deformation. The measuring ranges of the monitoring elements limit the researches on microstructure of the rock and soil slope failure mechanism. And the measuring range of the loading device limits the researches on the extreme problems.

Virtual numerical tests have advantages of modeling theoretical mathematical mechanics boundary conditions and random monitoring site and variables. The test could be carried out in great amount with idealization loading conditions and monitor objectives. It then brings convenience and economic benefit which is good for analyzing the slope failure systematically.

It is difficult for physical model experiment to control the loading conditions and the monitor site and variables veritably with limited techniques. Monitoring site and variables can hardly be random and the accuracy is subjected to the sensor accuracy and measuring range. The monitoring often is apparent. All these limit the researches on microstructure of the rock and soil slope failure mechanism.

Based on physical model experiment theories, the software established the numerical model of the rock and soil slope by mixing Matlab with fish language in the software platform of particle flow code (PFC). By programming loading code to simulate the centrifugal loading situation and monitoring program to supervise the stress, strain, displacement, velocity and strain rate of optional position, the software can analyze the basic slope failure mechanism and can be extended to a virtual test platform for other geotechnical engineering problems.

Functions and programming process

Main functions of the software include: (1) numerical model establishing for rock or soil slope with arbitrarily shape, the natural failure information increasing or decreasing. (2) procedural control of centrifugal loading or unloading conditions with optional order range. (3) loading and monitoring the natural damaged rock and soil slope, modeling the slope centrifugal model test.

The framework of the program is illustrated in figure 1. 


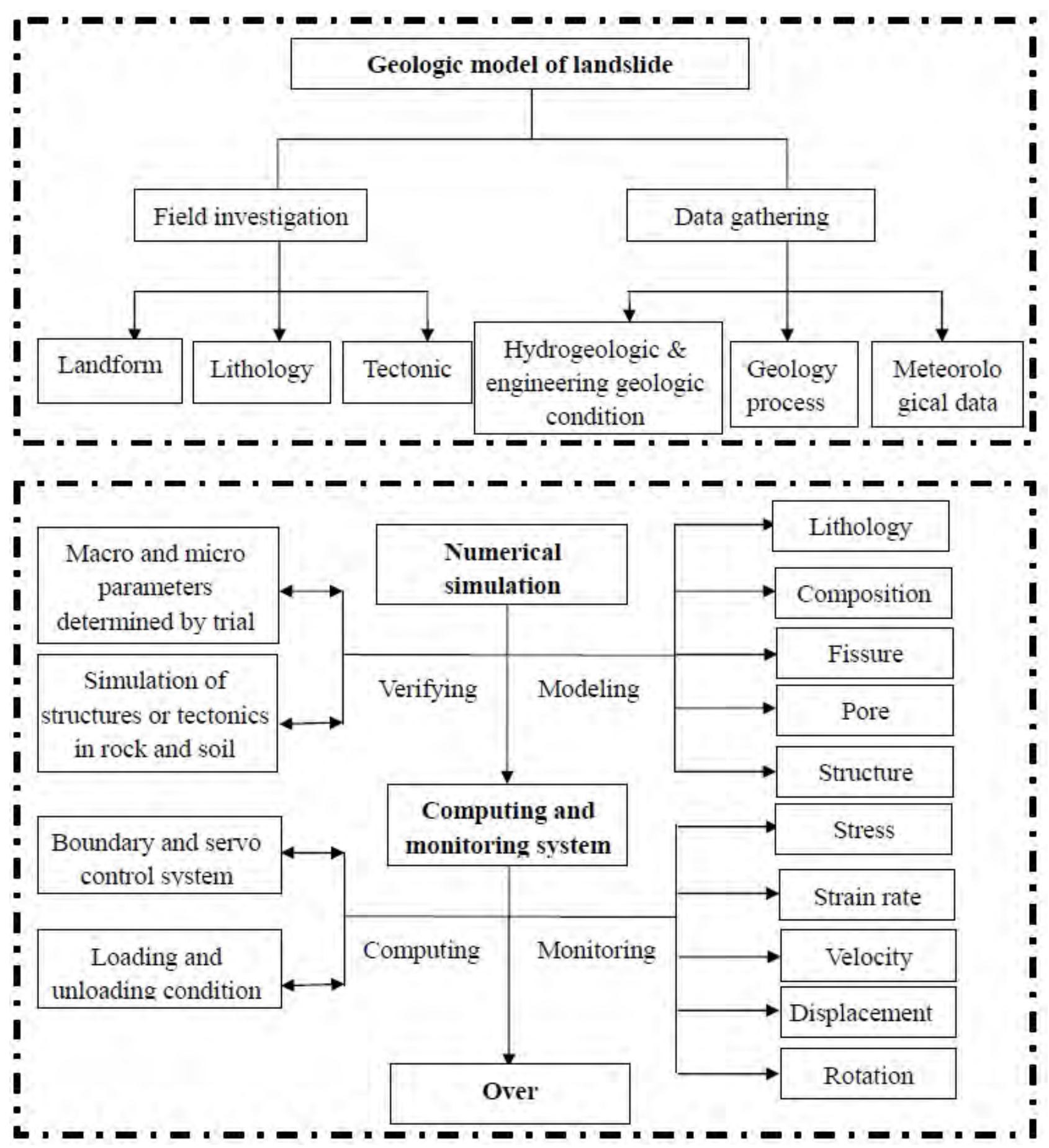

Fig.1 Program flow chart of the rock and soil mechanics virtual

\section{Program implementation and applications}

\section{Simulation of the relationship between the macro and meso parameters}

The PFC method is based on the mesoscopic parameters which are different from the macroscopic ones in the limit equilibrium method and normal finite element calculations. To make the analysis have same physical conditions and obtain the macroscopic parameters, compression tests of different strata with same size under $0 \mathrm{kPa}, 20 \mathrm{kPa}, 50 \mathrm{kPa}$ were carried out( with examples). The size of the specimen (same as in the slope model) is shown in figure2 (a). Figure2 (b) shows the compression curves. The peak values are $57.5 \mathrm{kPa}, 101.9 \mathrm{kPa}$ and $178.0 \mathrm{kPa}$, respectively. Three Mohr circles corresponding with the confining pressure are shown in figure2 (c). The relationship between the macro and meso parameters then can be established. 


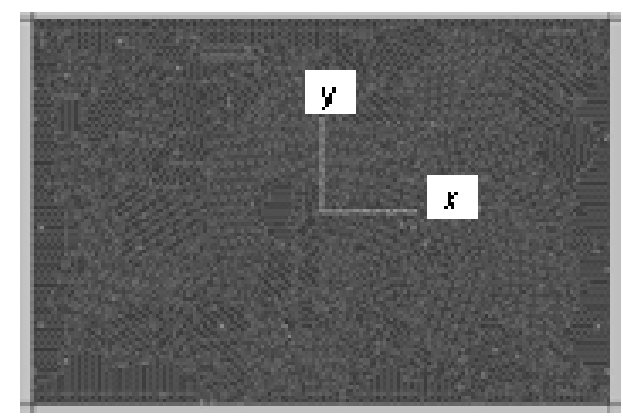

(a) Initial model

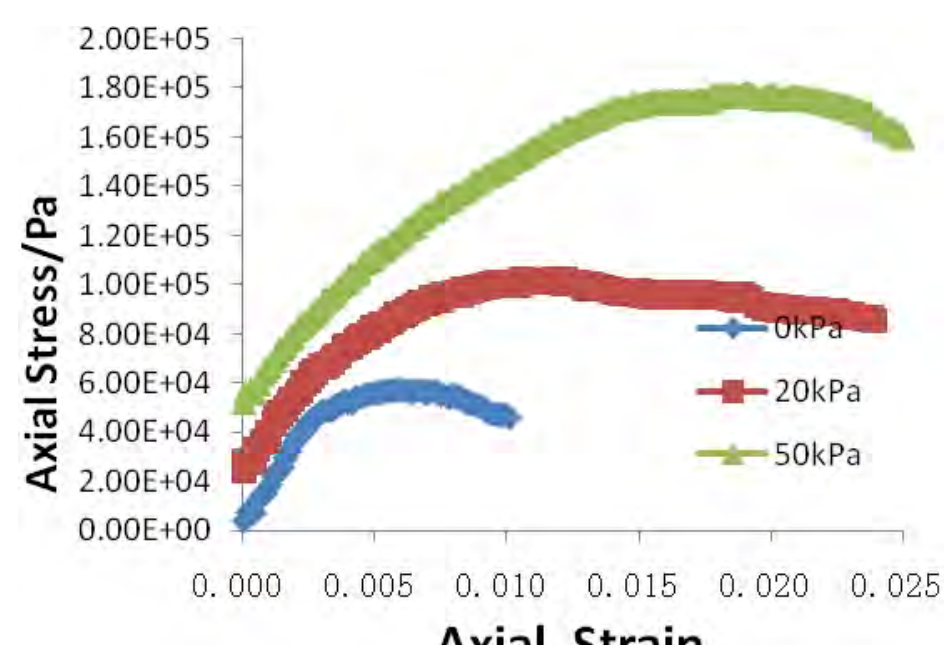

(b) Compression curves

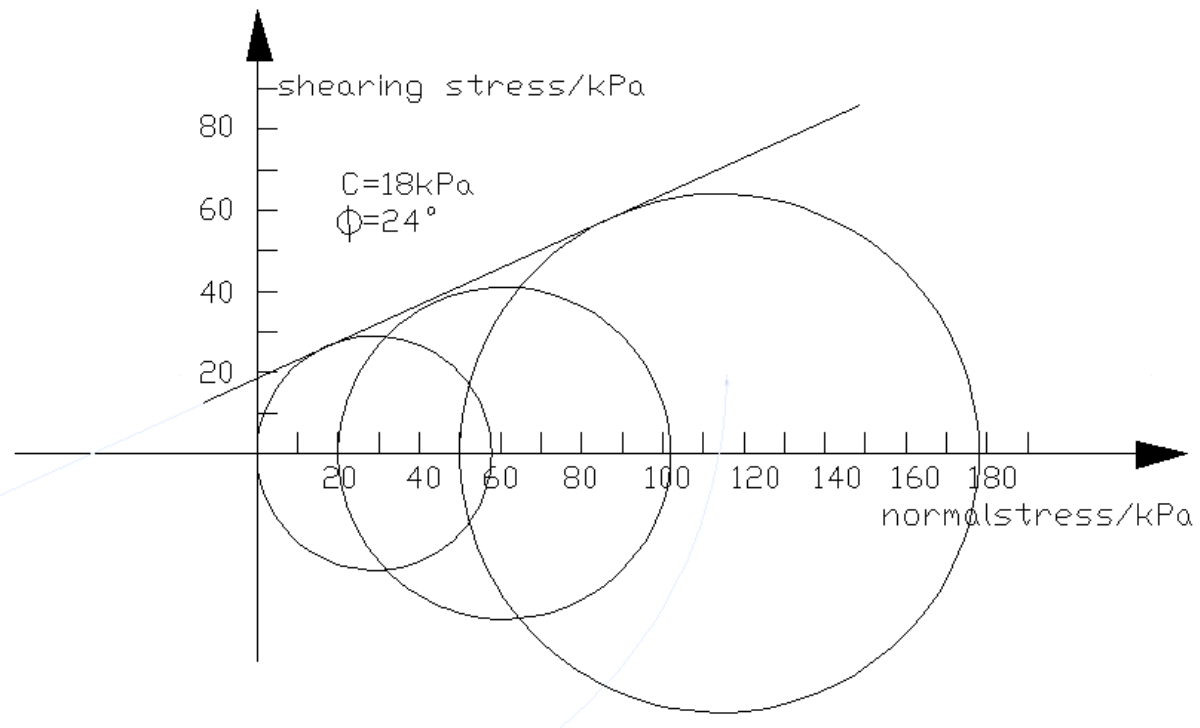

(c) Mohr-Column circle

Fig.2 Relationship between macro and meso parameters

\section{Simulation of slope problem}

Granule characteristics of different rock and soil can be analyzed by the particle curves and the calculation efficiency and simulation elaborate degree can be balanced. It should be pointed out that the difference between rock and soil is the cohesive strength of the particles. Both of them are particle congeries and form strata information and final different slopes by this structure.

Figure 3shows the simulation process as follow. The model size is $0.1 \mathrm{~m}, 30 \mathrm{~m}$ in width, $20 \mathrm{~m}$ in height. A monitor line is set at $\mathrm{x}=-5.0$, monitoring the slope down to $\mathrm{y}=-6.0 \mathrm{~m}$. The measuring 
sphere is $0.4 \mathrm{~m}$ in radius and monitors the stresses at $\mathrm{x}$ and $\mathrm{xy}$ directions. The interaction constitutive relation of the particles is cohesive model with unit weight $18.50 \mathrm{kN} / \mathrm{m} 3$, elastic modulus $15.5 \mathrm{MPa}$, friction coefficient 0.36 and cohesive strength $36.0 \mathrm{kPa}$. 30 vertical groups with $1.0 \mathrm{~m}$ interval are used to monitor the developing process of the slope sliding surface.

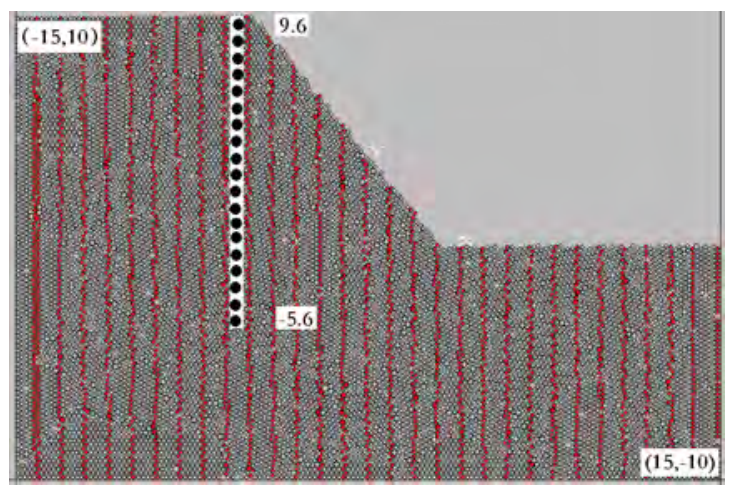

(a) Numerical model

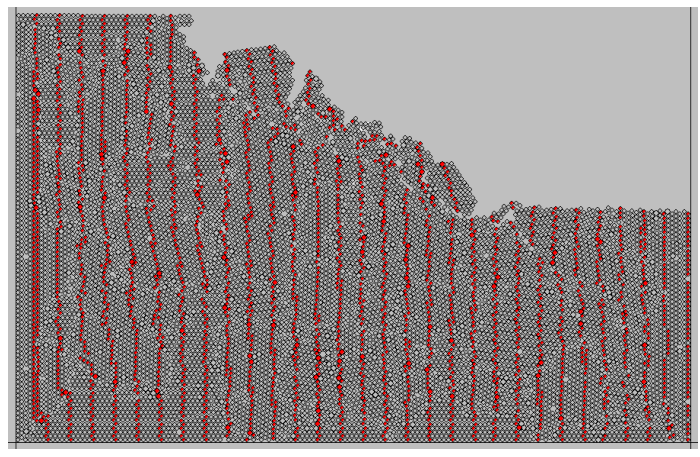

(b) 200,000th step fracture mode

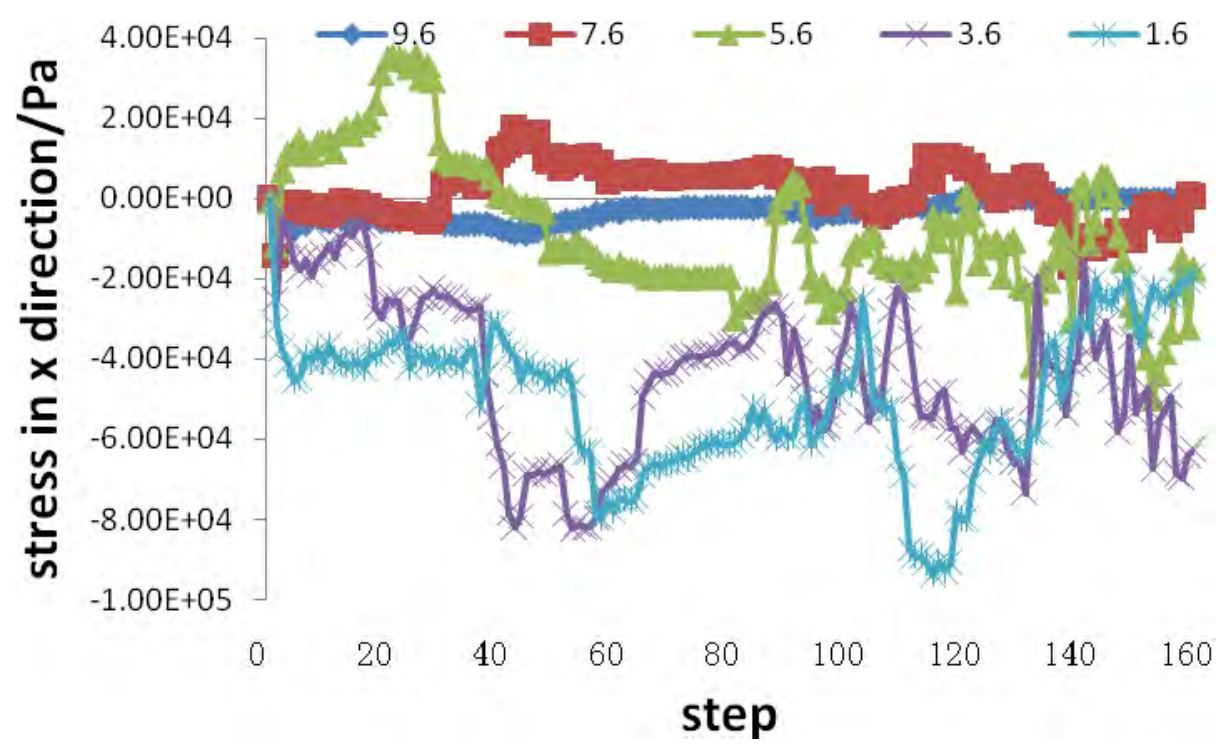

(c) Strain vs. time curve in $\mathrm{x}$ direction

Fig.3 Numerical model test result of slope problem

\section{Simulation of slide problem}

First of all, the difference between slide and slope is the former one has fixed slide surface and the latter one has potential slide surface which is unfixed. The modeling of slide surface can be carried out by constraining the slide mass with slide bed or by setting parameters of the slide soil. The first way was adopted in this paper. 
Establishing of the slope model and the monitoring function are shown in figure4. Degradation of the soil parameters and the subsequent slide initiating process with different water content in the lab were simulated. This process reflects indirectly the critical precipitation of different soil properties. In clay district, soil cannot saturate completely because of small permeability coefficient and the slope failure may happen for the volume weight increasing of certain thickness soil layer.

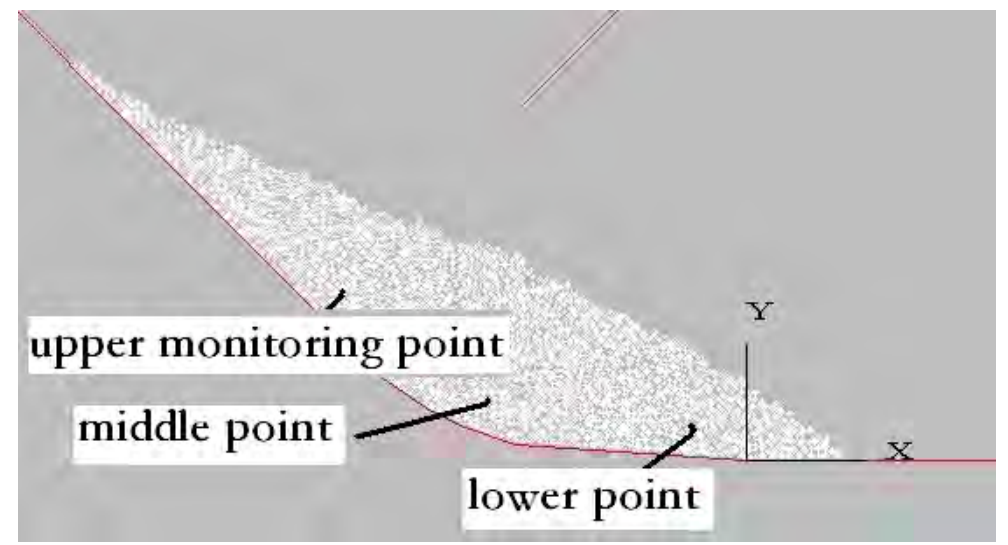

(a) Numerical model

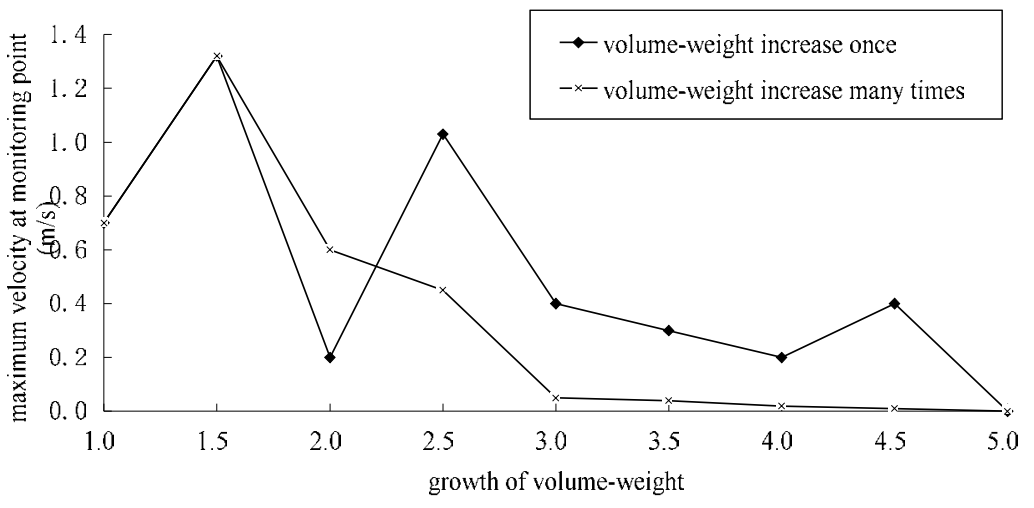

(b) Volume-weight \& maximum velocity curves

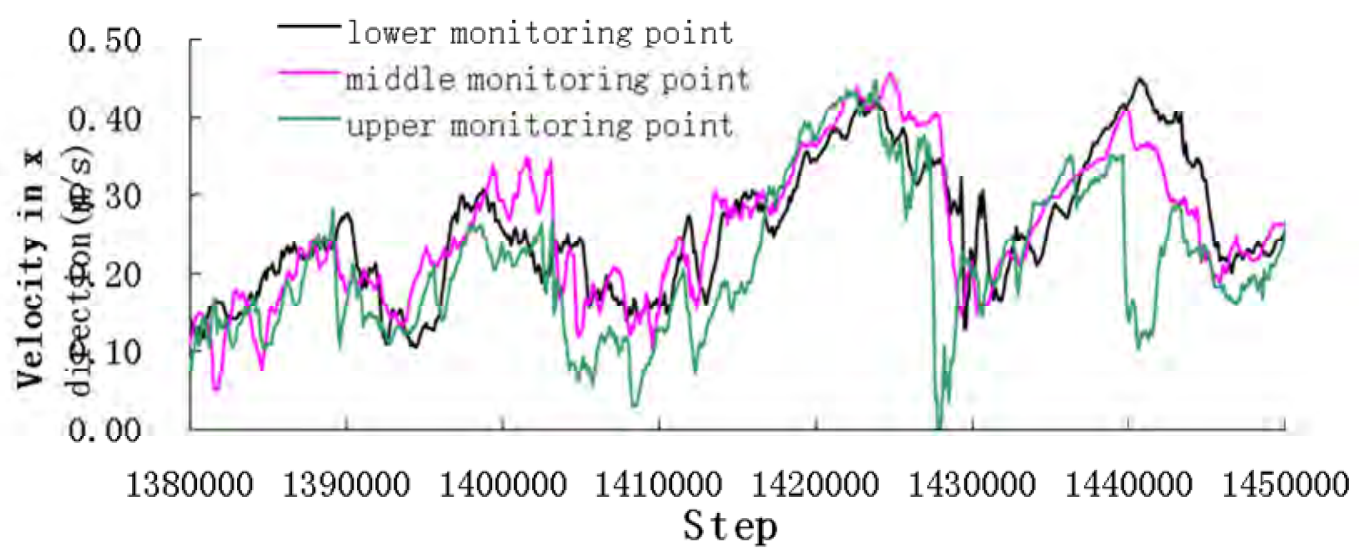

(c) Velocity fluctuation curves in $\mathrm{x}$ direction of different points

Fig.4 Numerical model test result of landslide problem

\section{Simulation of collapse problems}

Geology determination of collapse: comparing to slide, collapse mass has no distinct slide surface, the deposit structure is scatter and often conical. The topographic slope causing by the collapse usually is more than 45 degree with great altitude difference. The main terrain is isolate mountain spur or concave slope. Inner fractures develop in the slope, especially in the perpendicular and parallel directions of the slope. Tension fractures grow at the top of the slope and incise the 
slope. Fissures are about to connect each other and separate the mountain. In front of the slope there is free face or collapse mass which states the collapsing.

Figure 5 shows the failure modes of upright slopes caused by different parameter weakening ways. It turns out that the failure tends to be collapse mode and the slope wash has big volume with high rock and soil strength, especially with high cohesion. While with low strength the failure tends to be slide mode. With constant cohesion internal friction angle cannot dominate the failure. The cohesion has crucial impact on the upright of the rock and soil mass. The internal friction angle can control the stable angle of the incompact pile slope.

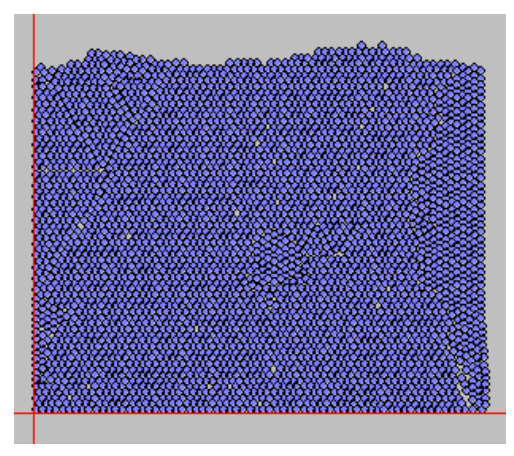

(a) Initial model

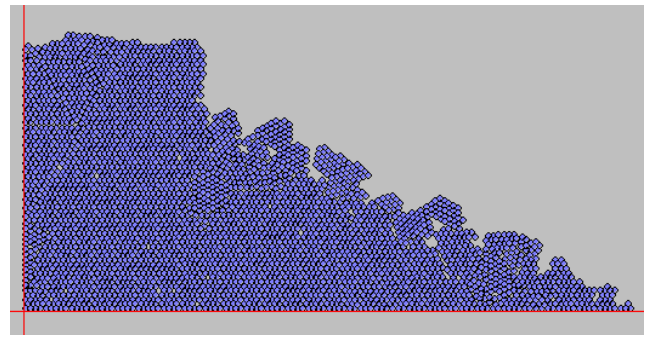

(b) Final collapse mass

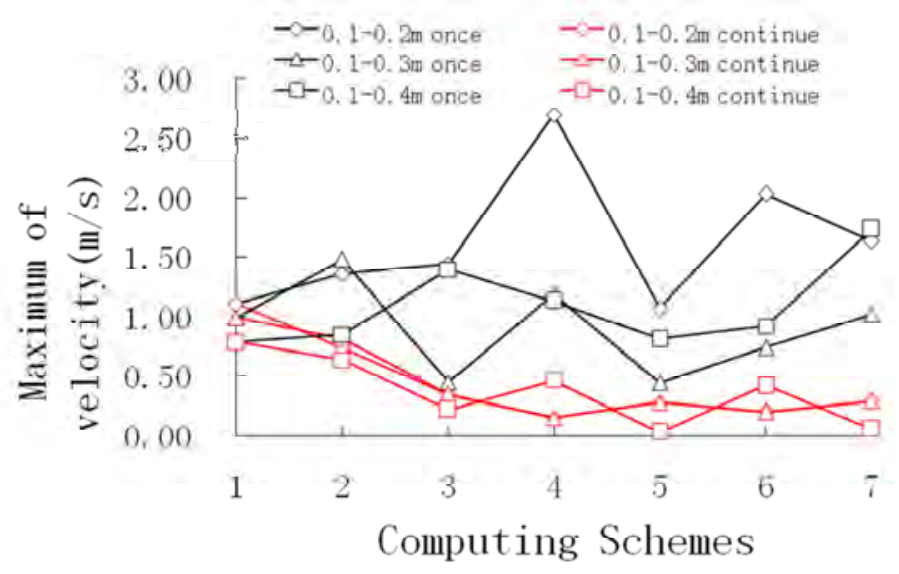

(c) Maximum velocity curves of different reduction methods

Fig.5 Numerical model test result of collapse problem

\section{Simulation of the slopes problems concerning with material compositions and structure characteristics}

Besides the former simulation problems, the simulation concerning with material composition that has great effect on the gravelly soil slope is also important. This kind of failure is related to not only the strength but also the granular and particle composition. By constructing various clump the aggregate cracking, i.e., gravel breaking can be simulated. Hitherto the uniform gravel, graded gravel and gravel with various structures can be modeled by the method. 
With constant number and position, increased size is good for stable aggregate framework and will impede local deformation and that both facilitate the slope stability. Figure6 (b) shows three shear stress curves. Change of the shear stress tends to be steady with the gravel size increasing which improved the force sustaining forms of the slope. The result shows that the gravel size investigation and analysis should be added to the reconnaissance of gravelly soil slope.

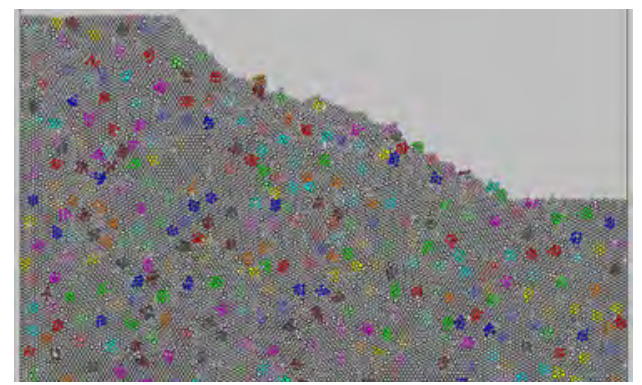

(a) $\mathrm{r}=0.3 \mathrm{~m}$

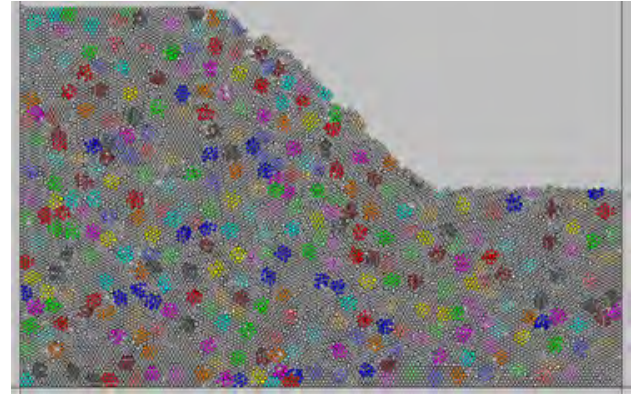

(b) $\mathrm{r}=0.4 \mathrm{~m}$

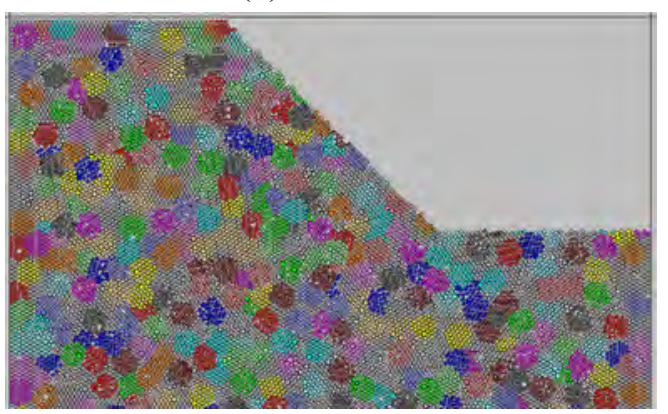

(c) $\mathrm{r}=0.6 \mathrm{~m}$

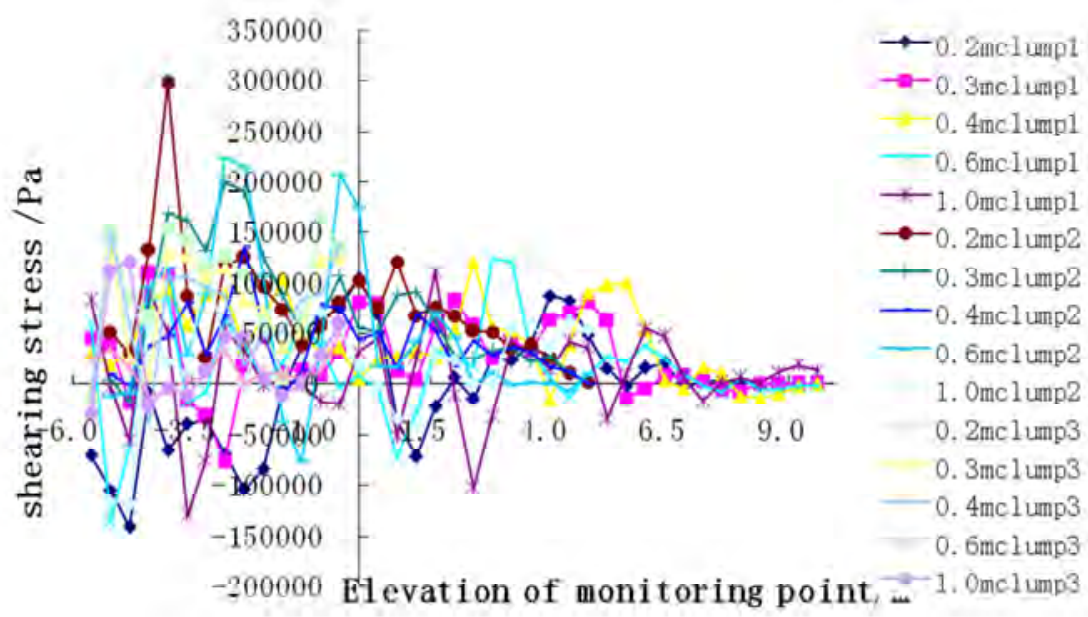

(d) three shear stress curves

Fig.6 Final shape of gravel soil slope with different size of grain and same grain group under $6 \mathrm{~g}$ loading condition 
Figure 7(a) shows different failure forms caused by different joints. Figure 7(b) shows the slope failure caused by unloading fracture of the Longjiang super bridge.

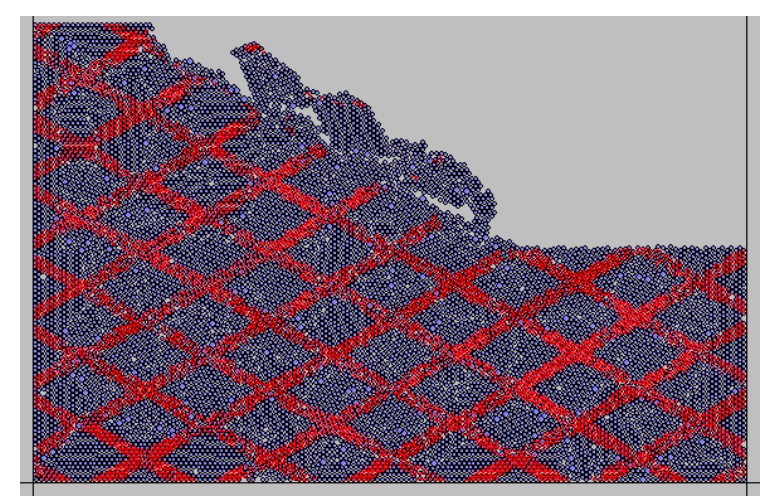

(a) Two sets of joints

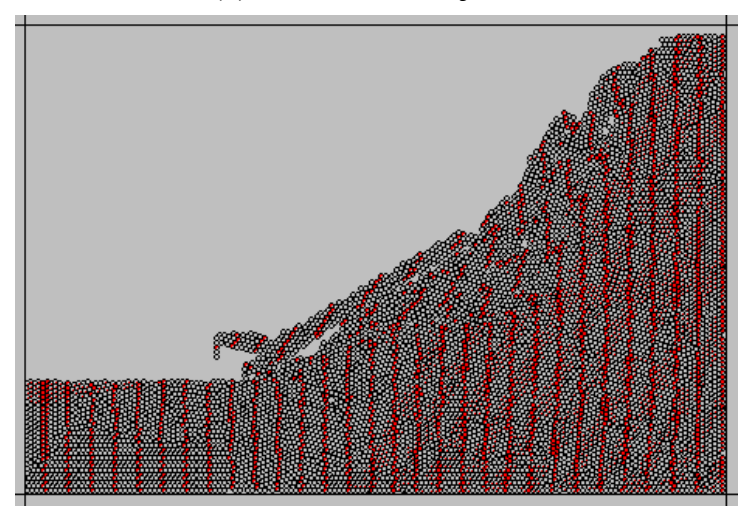

(b) Unloading fractures in different depth

Fig.7 Final failing shape of slope affected by joints and unloading fracture

\section{Conclusions}

The program realized all the functions for slope and slide virtual test model and supplemented some monitoring objects including inner energy evolution and micro crack developing. These results are significant for the study of the impact of natural structure of the geotechnical materials and its macro and meso mechanics on the slope and slide failure mechanism.

\section{Acknowledge}

This work was financially supported by the National Basic Research Program of China (973 Program) (2011CB013505) and China academy of sciences knowledge innovation program (No. kzcx2-yw-151).

\section{References}

[1] Chen Zuyu. Soil slope stability analysis: theory, methods and programs [M]. Beijing, China water and power press. 2003:1-5.

[2] Chen Zuyu. Rock slope stability analysis: theory, methods and programs (2nd edtion) [M].

Beijing, China water and power press. 2005:640-652.

[3] LIU Yanzhang,GE Xiurun,LI Chunguang,et al.Stability analysis of slope and dam foundation based on vector method safety factor[J]. Chinese Journal of Rock Mechanics and Engineering,2007,26(10): 2 130-2 139.(in Chinese) 
[4] LI Chun-guang,ZHUYu-fei,LIU Feng et. Al. Evaluation of strength reduction factor by lower bound limit analysis using finite element method[J],Rock and Soil Mechanics,2012,Vol.33(6): 1816-1821.

[5] ZHENG Hong,LI Chun-guang,LEE C F,et al.Finite element method for solving the factor of safety[J]. Chinese Jounal of Geotechnical Engineering,2002, 24(5):626-628.

[6] LU Qing,SUN Hongyue,SHANG Yuequan.Slope failure criteria of shear strength reduction finite element method[J].Journal of Zhejiang University: Engineering Science,2008,42(1): 83-87.(in Chinese)

[7] XU Weiya,XIAO Wu.Study on slope failure criterion based on strength reduction and gravity increase method[J].Rock and Soil Mechanics,2007,28(3): 505-511.(in Chinese)

[8] LUO Xianqi,GE Xiurun.Theory and application of model test on landslide[M]. Beijing: China

Water Power Press,2008: 84-92.(in Chinese)

[9] REN Wei-zhong, CHEN Hao. Model testing research on deformation and fracture mechanism of landslide and its harnessing engineering $[\mathrm{J}]$.Chinese Journal of Rock Mechanics and Engineering,2005, 24(12): 2136-2141. (in Chinese)

[10]SUN Guan-hua,ZHENG Hong, LI Chun-guang. Searching critical slip surface of three-dimensional slopes based on equivalent plastic strain[J].Rock and Soil Mechanics, 2010, 31(2):627-632. (in Chinese)

[11] LU Kun-lin,ZHU Da-yong,YANG Yang. Model test study of slope failure progress[J],Rock and Soil Mechanics,2012,Vol.33(3):778-782. (in Chinese)

[12]DONG Jinyu,YANG Guoxiang,WU Faquan,et al.The large-scale shaking table test study of dynamic response and failure mode of bedding rock slope under earthquake[J].Rock and Soil Mechanics,2011,32(10): 2 977-2 983.(in Chinese) 